\title{
Logiques Et Mécanismes Endogènes De Conservation Et De Protection De La Biodiversité Chez Les Agni-Bini A L'est De La Côte D'ivoire
}

\author{
AFFESSI Adon Simon, KOFFI Koffi Gnamien Jean-Claude, ADINGRA \\ Tano Kouadio, \\ Membre du Laboratoire d'Etudes et de Recherches Interdisciplinaires en Sciences Sociales(LERISS), \\ Université Félix Houphouët-Boigny d'Abidjan
}

\begin{abstract}
Résumé :- Le recours aux pratiques liées à la conservation endogène du couvert végétal est pour l'ONU, les écologistes et les scientifiques, une alternative crédible face à l'érosion accélérée des ressources naturelles, au dérèglement climatique et à la prolifération du taux de $\mathrm{CO}_{2}$ dans l'atmosphère. Cette étude montre les mécanismes endogènes de conservation de la biodiversité chez les Agni-Bini. Pour traiter cette question, l'étude s'est déroulée dans neuf (9) villages et deux(2) campements des sous-préfectures de Kouassi-Datékro et Boahia à l'Est de la Côte d'Ivoire. Des guides d'entretien ont été adressé aux autorités coutumières, aux représentants des différentes structures sociales, aux chefs des communautés religieuses, à des allogènes et des allochtones. Les méthodes historique, dialectique et fonctionnaliste ont été mobilisées dans le traitement des informations. L'étude a montré que l'idéologie religieuse traditionnelle africaine fondée sur une alliance individu-nature ou même communauté-nature, une alliance étroite entre les dieux et la nature est le fondement de la protection et de la conservation de l'environnement. La subsistance de cette alliance confirme donc une relation de symbiose entre l'Agni-Bini et son patrimoine naturel.
\end{abstract}

\section{Mots clés : environnement-conservation endogènes-biodiversité-ressources naturelles.}

\begin{abstract}
To rely on practices related to the endogenous conservation of the vegetation cover is according to the United Nations, ecologists and scientists a credible alternative to face the accelerated erosion of natural resources. And it is also a credible alternative to face climate change and to cope with the proliferation of $\mathrm{CO} 2$ in the atmosphere. This study shows the endogenous mechanisms of biodiversity conservation by the Agni-Bini community.To deal with this issue, the study was carried out in nine (9) villages and two (2) camps, all located in the sub-prefectures of Kouassi-Datékro and Boahia in the East of Ivory coast (Côte d'Ivoire).The guides of interview have been sent to traditional authorities, to representatives of the different social structures, to leaders of religious communities, and also to natives, non-natives and foreigners. Historical, dialectical and functionalist methods have been involved and used in the processing of information. The study has demonstrated that the traditional African religious ideology founded on the relationship individual-nature or as well as the relationship community-nature, meaning also a close relationship between the gods and the nature is indeed the real foundation of the protection and conservation of environment. The subsistence of such a relationship confirms therefore a symbiosis between the Agni-Bini community and their natural heritage.
\end{abstract}

Keywords: environment, endogenous conservation, biodiversity, natural resources.

\section{I.}

\section{.INTRODUCTION}

A l'indépendance, la Côte d'Ivoire avait un couvert végétal estimé à 16 millions d'hectares. Aujourd'hui, il ne compte que 3 millions (Ibo, 2004). Cette situation montre le dépérissement accéléré de la forêt malgré toutes les actions de protection et de sauvegarde du couvert végétal exécutées par l'Etat. En effet, la nécessité de protéger la diversité biologique n'est pas récente. Elle est constatée chez plusieurs peuples. Ainsi, les sociétés humaines quelque soit leur niveau d'évolution ont toujours mis en œuvre des mécanismes de manière endogène ou par emprunt de réguler leur relation avec les milieux qui les portent (Ibo, idem). L'on comprend que l'homme est une entité indissociable de la nature. En d'autres termes, entre l'homme et la nature il n'existe qu'une différence de degré et non une différence de nature. Ainsi, pour l'africain, toute action destructrice sur la nature, détériore gravement les conditions de vie de l'homme. Concernant laCôte 'Ivoire, la gestion des ressources forestières a été amorcée par le colonisateur avec la mise en place depuis 1926, des deux premiers parcs naturels de son histoire, à savoir le parc de la Comoé en zone de savane et celui de Taï en zone forestière. En 1960, il a été institué un Comité National de la Protection de la Nature (CNPN) ayant pour principale attribution la création de réserves naturelles (Ibo, idem). Aussi, en 1966, la Société pour le Développement des Forêts (SODEFOR) fut- elle créée, constituant ainsi une étape importante dans le processus 
de consolidation du cadre institutionnel du secteur forestier. Le mode de gestion des ressources naturelles ivoiriennes essentiellement basé sur les méthodes modernes de gestion, a été emprunté aux civilisations occidentales. Ce sont des méthodes qui mettent en place des mécanismes rigides (présence des eaux et forêts) de gestion.Par ailleurs, en plus des agents des eaux et forêts chargés de la surveillance des espaces protégés, des actions traditionnelles de protection ont existé dans chacune des cultures du peuple ivoirien. Il s'agit des totems, des tabous et des interdits qui s'appliquent aux espèces animales et ligneuses ainsi qu'aux espaces naturels instaurés pour exploiter de façon rationnelle les ressources naturelles. Les Agni-Bini ne sont pas en marge de ces pratiques. Ils observent aussi bien ces différentes pratiques dans leur environnement social. En effet, chez ce peuple, l'abattage de certains arbres, la prohibition formelle de fréquenter des sites dits sacrés et la consommation de certains animaux sont strictement interdits. Quelles sont les logiques locales qui sous-tendent la protection des sites et des espèces chez les Agni-bini de Côte d'Ivoire ?Pourquoi observe-t-on des mesures de protection de sites et d'espèces ?Quelles sont les logiques locales qui sous-tendent la protection des sites et des espèces chez les Agni-bini de Côte d'Ivoire ?Quelles sont les fonctions que jouent ces espaces et espèces ligneuses ?Quels sont les facteurs explicatifs de la survivance des pratiques traditionnelles de protection?

\section{METHODOLOGIE}

L'étude s'est déroulée dans neuf (09) villages et deux(2) campements des sous-préfectures de KouassiDatékro et Boahia à l'Est de la Côte d'Ivoire Elle a débuté dans la période de Novembre 2015 et a pris fin dans le mois de Juin 2016 de façon discontinue. L'enquête s'est réalisée respectivement dans les villages suivants : Kouassi-Datékro chef lieu de sous-préfecture, Komambo, Abondji-Morokro, Kotronou, Yakassé-Bini, KouassiBilékro ainsi que les campements Bomirenou et Konoman affiliés à la sous-préfecture de Kouassi-Datékro. Dans la Sous-préfecture de Boahia, le chef lieu de sous-préfecture et les villages de Domorossi et Daboyakro ont été retenus. Le choix de ces villages se justifie par la présence effective des espèces et espaces protégés. L'approche qualitative et l'entretien semi- directif ont été mobilisés dans l'optique de mieux cerner les questions en lien avec les logiques sociales de protection des sites et des espèces et les fonctions que jouent ces espèces et espaces dans l'arène sociale du peuple agni-Bini. Au total soixante trois (63) acteurs ont été interrogés dans les principes de la saturation des données et répartis comme suit : 12 autorités coutumières, 6 personnes membres des structures sociales, 13 personnes de la communauté religieuse, 20 allogènes détenteurs du savoir-faire, du savoir-être et gardiennes des valeurs culturelles ancestrales, 12 allochtones. De plus de ces entretiens, il a été réalisé une observation participante sur quelques sites et espèces pour prendre connaissance des espèces et espaces. Une prise de vue des différents éléments observés a été faite à partir de cette observation.Les données collectées auprès des différentes catégories susmentionnées ont fait l'objet d'analyse par le recours à trois méthodes. La méthode historique est utilisée pour appréhender les ressorts historiques de toutes les interdictions liées au prélèvement des ressources naturelles et cerner les origines des totems, des modes d'organisation politiques, économiques et sociales hérités par ce peuple avant la colonisation. La méthode dialectique a servi de comprendre les contradictions, les ambivalences, les incohérences mais également la cohésion et l'organisation sociale de ce peuple. Enfin, la méthode fonctionnaliste a contribué à saisir les fonctions que remplissent les espèces et espaces dont l'usage et la consommation sont proscrites. En clair ces méthodes ont été utiles pour comprendre les fondements des logiques et pratiques qui sous-tendent la protection des espaces et espèces chez les Agni-bini de Côte d'Ivoire.Pour l'analyse des données, l'on a procédé à la retranscription intégrale des entretiens réalisés avec les différents acteurs puis à une catégorisation des idées. A partir de cette retranscription, une analyse du contenu du discours des enquêtés a été réalisée.

\section{RESULTATS}

\section{I- NOMENCLATURE DES ESPACES ET ESPECES PROTEGES PAR LE PEUPLE AGNI-BINI 1-1 les espaces protégés}

Il s'agissait de sites bien protégés et n'y pénétraient que les individus possédant un certain pouvoir mystique (féticheurs, chasseurs et guérisseurs). En dehors de ces initiés, ces sites sont interdits d'activités anthropiques. Ces espaces sont riches en espèces fauniques et floristiques. Il s'agit en effet de la forêt de la colline Mérékou de Konoman, de la forêt qui entoure le marigot "assiédou n'zuéba" situé entre KouassiDatekro et Yakassé-Bini, de la forêt de gnan-kotoua de Kouassi-Bilékro, des forêts loyebooh et mansèh à Abondji- Morokro sans oublier la forêt galerie de la rivière N'Djoré qui arrose pratiquement les deux souspréfectures.Dans les villages enquêtés, il existe plusieurs massifs forestiers allant de trois (03) à cinq(05) hectares qui sont interdits d'accès aux non initiés. Les dénominations de ces formations végétales ont la particularité de ne rien signifier la plus part du temps dans la langue locale. Répondant à notre préoccupation à ce sujet un notable de KOUASSI-BILEKRO nous a révélé que « ces noms sont ceux des génies dépositaires des lieux». Ces sites sont en général des lieux de pèlerinage pour les populations animistes en vue d'expier leurs péchés et d'offrir des sacrifices aux mânes et esprits bienfaiteurs pour améliorer leurs conditions de vie 
sociales.Il s'agit de la forêt sacrée 'Mansêh'” de Kotronou qui a en son sein une pierre du même nom que les populations vénèrent. A Abondji-Morokro une autre forêt portant le même nom et protégeant une autre pierre du même nom. A la faveur de nos investigations sur la forêt sacrée de Kotronou le vieux Andji Y. a indiqué que «les deux forêts sont d'origines différentes. En effet, deux personnes ont eu la révélation de la puissance des pierres à des moments différents. Selon le site, chaque pierre possède un pouvoir spécifique. Ici à Kotronou la pierre de la forêt sacrée protège les populations du village des épidémies et des malheurs qui pourraient les frapper $\gg$.

A Abondji-Morokro, Nanan Yao. G; chef du village d'Abondji nous a révélé que "la pierre qui se trouve dans la forêt concède à tout individu qui le souhaite, prospérité et gloire en contrepartie d'offrande cathartique ».

\section{1-2- les cours d'eaux sacrées}

A l'image des forêts, les cours d'eaux sacrées existent depuis la création du village et institués comme tel par l'ancêtre éponyme. Leur consécration comme des espaces protégés s'explique par les services rendus à des moments donnés à cette communauté. Aussi, les interdictions liées aux actions anthropiques sur ces écosystèmes procèdent de la présence d'esprits (djinan) qui les habitent. Ainsi, profanés ces plans d'eau suscitent la colère des locataires de ces écosystèmes. Ceux-ci, pour se venger des contrevenants, peuvent leur jeter de mauvais sorts (maladies, déformations physiques, infertilité...).Ces cours d'eau sont pour l'essentiel composés de marres, de sources et de marigot. Certains membres de la communauté peuvent les fréquenter pour y puiser de l'eau. Cependant, il existe d'autres cours d'eaux dont l'accès n'est autorisé qu'aux seuls initiés. Toutefois, il convient de noter que pour ce qui concerne les points d'eau fréquentables, les femmes ayant déjà procréées n'y ont pas accès les jours 'noirs', (les fô et les djinigo jours de repos obligatoire). L'activité de pêche y est formellement interdite et les massifs forestiers environnants ne doivent être ni défrichés encore moins y mener une quelconque activité cynégétique. Il en existe plusieurs dans cette région. L'on peut citer parmi ceux-ci le ''Djorodjoro', cours d'eau sacré portant le nom du village dont les populations vénèrent, la source d'eau " Nandôh' et le marigot bgèrè à KOTRONOU, le 'Kromida'" du campement BOMIRENOU et "'l'assedou n'zueba" de KOUASSI-DATEKRO. Le cours d'eau de 'Kromida' situé à 22 kilomètres de KOUASSI-DATEKRO ne tarit jamais quelle que soit l'avancée de la sécheresse. Avant l'installation de la pompe villageoise, le 'Kromida' le était la seule source d'approvisionnement des femmes en eau pour satisfaire les besoins de la communauté. Cette eau est riche en poisson et est interdite de pêche. En effet le non respect de cet interdit par un individu converti au christianisme à travers une activité de pêche a causé sa mort. Etant dans l'eau au cours de la pêche, il a reçu une piqure sur l'index droit qu'il n'a révélé qu'au moment où, une fois à l'hôpital, tout son côté droit s'était tuméfié de façon incommensurable. Il a par la suite perdu la vie au lendemain de son internement à l'hôpital. Pour implorer la clémence des esprits et prémunir la progéniture de l'infortuné du courroux des génies habitant ce site, des sacrifices expiatoires et propitiatoires ont été faits par le chef de terre. La mésaventure de cet infortuné a accru davantage au sein de la communauté le strict respect et la crainte de cette eau sacrée.

\section{1-3- arbres sauvegardés}

L'agriculture est l'activité principale des Agni Bini. Cette activité nécessite le défrichage de l'espace à cultiver sans oublier l'usage de la technique de brûlis. Ces méthodes, singulièrement, la technique de brûlis conditionnent une bonne récolte. C'est ce que nous raconte l'un de nos enquêtés dans la sous- préfecture de KOUASSI-DATEKRO en ces termes : «Autrefois mon grand-père m'a révélé qu'à la suite d'une discussion sur la fertilisation du sol par la technique de brûlis; deux vieux ce sont proposés de faire l'expérience. A la saison de la mise en culture, l'un a fait son champ sans en avoir eu recours. L'autre par contre a utilisé la technique de brûlis. Au moment des récoltes, le vieux qui a utilisé la technique de brûlis a eu son grenier beaucoup plus fourni que l'autre. Depuis cette époque, la population a donc toujours utilisé la technique de brûlis ». Si une telle technique augmente la production agricole, il faudrait y prendre garde dans son usage en songeant à protéger certaines espèces végétales se trouvant sur l'espace à cultiver. Ces espèces devront être préservées au moment de la préparation des espaces pour la mise en cultures. Il s'agit d'un certain nombre d'arbres que sont : «le koto », «le tchimi », «le walè» le fromager « gnanhan », le néré « n'drè », l'iroko «fondo » le «lôuye », le lèn'guè et le «nahi ». Ces espèces sont interdites de coupe. Il est formellement proscrit de faire du feu sous ces arbres sous peine de sanctions.

Tableau 1 : espèces ligneuses conservées chez les Agni-bini

\begin{tabular}{|l|l|l|l|l}
\hline Figures & Noms & Familles & Noms français & Noms scientifiques \\
\hline
\end{tabular}




\begin{tabular}{|l|l|l|l|l|}
\hline & locaux & & & \\
\hline 1 & Walè & Sterculiacea & - & Cola gigantea \\
\hline 2 & Tchimi & Ebenaceae & $\begin{array}{l}\text { Ebenier } \\
\text { d'Afrique }\end{array}$ & Diospyros mespiliformis \\
\hline 3 & Gnanhan & Bombacacea & Fromager & Ceiba pentandra \\
\hline 4 & Fondo & Moracea & Iroko & Chlophora excelsa \\
\hline 5 & Koto & Sterculiacea & Prunier noir & Vitex doniana \\
\hline 6 & N'drè & Mimosacea & Néré & Parkia biglobosa \\
\hline 7 & Lèn'guè & Fabacacea & & Daniellia Oliveri \\
\hline 8 & Lôye & Caesalpiniaceae & Lim du Gabon & Erythrophleum ivorense \\
\hline 9 & Nahi & Mimosacea & Dabéma & Piptadeniastrum africanum \\
\hline
\end{tabular}

Source : notre enquête, 2016

Figure 1 : Feuilles des arbres protégés par les Agni-Bini selon l'ordre du tableau 1
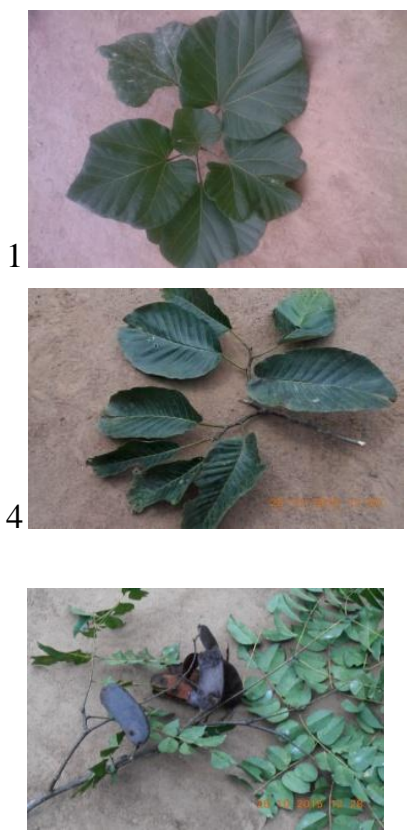

7
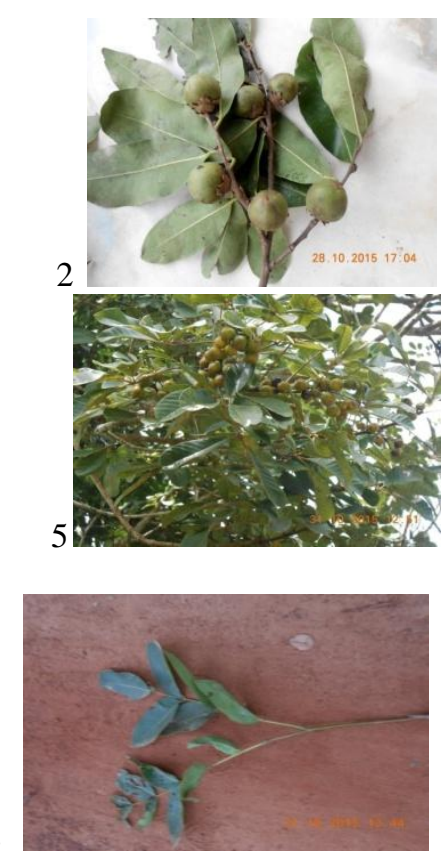

3

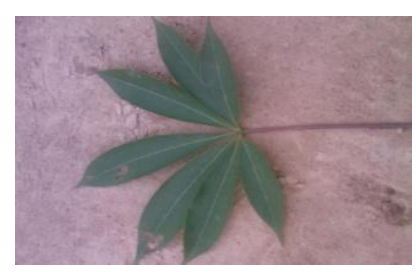

6

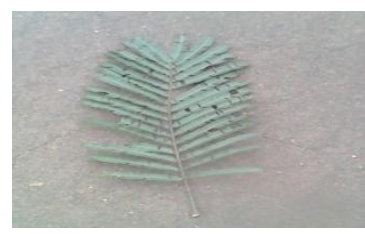

9

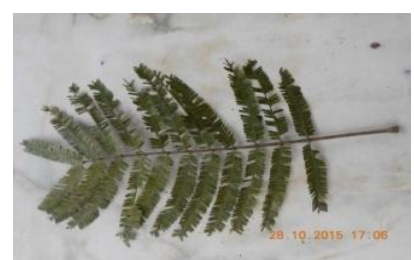

Source : notre enquête, 2016

\section{FONDEMENTS DE LA CONSERVATION DES PAYSAGES RURAUX \\ II-1- Origines de la conservation des couverts ligneux \\ II-1-1- Origines mystiques}

Les origines mystiques de la conservation des espaces et espèces s'expliquent par l'action des féticheurs et autres «comiens». En effet, avant la création de chaque village chez le Bini, les féticheurs font des sacrifices et exécutent des pas de danses prémonitoires pour rassurer les chefs de l'opportunité de leur projet. C'est donc eux, qui par des cérémonies discutent avec les génies (ils comprennent leur langage), propriétaires terriens qui autorisent ou non la création du village. Si ce projet est accepté, ils ne manquent pas de poser les conditions de cohabitation. C'est ainsi qu'ils préconisent que l'on protège tel ou tel espace et que l'on n'abatte pas tel arbre et tel autre.Notons que c'est en reconnaissance de leurs bienfaits (consommation des fruits, écorces et feuilles curatives) pour les génies, que ces arbres sont aujourd'hui interdits de coupe. Leurs utilités pour les esprits et les génies peuvent servir aussi aux hommes ; futurs habitants de cet espace. D'autres espèces ligneuses (fromager, iroko et Cola Gigantea (walè)) sont également conservées pour avoir été indiquées par des génies à des chasseurs comme leur lieu d'habitation. Ils ne voudraient donc pas être dérangés un jour pendant qu'ils se reposeraient. Ce sont des arbres craints. Toute action consistant à abattre ces ressources ligneuses expose l'individu à la sentence des génies et des mannes. 


\section{II-1-2 Origines culturelles et patrimoniales}

Les ressources ligneuses protégées par les Agni-Bini sont la propriété des génies et des ancêtres mais également de la communauté toute entière car, pour se nourrir et se soigner, ils ont recours aux racines, aux écorces et aux feuilles. La destruction de ses ressources engendre une menace pour la survie de ce peuple. Toutefois, elles sont très importantes dans la cosmogonie Agni pour l'homme et l'animal. En effet, selon les autochtones, gardiens des valeurs culturelles ancestrales enquêtés; ces arbres existaient avant la création des villages. S'ils subsistent encore aujourd'hui, c'est grâce à la rigueur de la gestion des ancêtres quand ils vivaient encore. Quand bien même ils ne soient plus de ce monde, ils demeurent encore regardant quant à la protection de ces espèces. Il en va de même pour les génies de la brousse qui comme les ancêtres ne transigent pas sur les actions destructrices de l'homme sur les espèces sauvegardées. Dans la mesure où, pour eux, par ces actions les vivants ont tendance à violer le pacte signé avec l'ancêtre éponyme à la création du village. L'imminence de la réprimande de ces divinités invisibles oblige les vieux, détenteurs de ces savoirs à veiller à la sauvegarde de ces ressources, laquelle sauvegarde garantie la survie même du peuple.

\section{FONCTIONS TRADITIONNELLLES DES ARBRES PROTEGES III-1-Fonctions médicinales}

La plupart des arbres protégés chez le peuple Agni Bini remplit des fonctions d'ordre médical. C'est ce qui justifie les prélèvements d'écorce sur les troncs du néré et du koto constaté pendant l'enquête de terrain. Suite à nos interrogations, un guérisseur nous répond en ces termes : "ces prélèvements d'écorces servent à soigner des malades. Les esprits qui guident les guérisseurs sont nombreux et chaque plante soigne selon les consignes de l'esprit et les incantations qui précèdent chaque prélèvement». Pour ce qui est des fruits de ces plantes, ils sont aussi utilisés à des fins thérapeutiques. C'est le cas de la moutarde obtenu à partir des grains du néré appelé kondrô en langue locale et plus connu en Afrique de l'Ouest sous le nom de soumara. Cette moutarde régulerait la tension artérielle. Quant au lait noirâtre obtenu à l'aide des fruits de koto bien mûrs, il faciliterait la digestion et lutterait contre les douleurs abdominales. La sève du fondô (Iroko) guérirait les plaies incurables. L'écorce de néré infusée à feu doux pendant un quart d'heure guérirait l'ulcère d'estomac. Les sites sacrés sont fréquentés par les devins et autres guérisseurs pour le prélèvement de certaines ressources qui n'existent que dans ces lieux à l'effet de concevoir des potions curatives.

\section{III-2- Fonctions alimentaires}

Une légende raconte que les arbres interdits de coupe, les arbres fruitiers pour la plupart : «ont servi autrefois de nourriture aux ancêtres quand une grande famine survînt. La poudre de néré par exemple a servi de lait pour les nouveaux nés orphelins de mère et le jus des fruits du "tchimi"' a fait office de sucre pour assaisonner la bouillie des nourrissons. Aussi, le lait obtenu à l'aide des grains de koto servait-il à nourrir les enfants et les malades. C'est pour ces raisons que l'on refuse qu'on les abatte. Il ne faut pas être ingrat » (propos du chef de terre de Daboyakro). Aujourd'hui encore, au moment des semis, les enfants ramassent les fruits de ces arbres pour les consommer comme des friandises. Les feuilles du koto et du gnanhan servent à faire de la sauce. Les animaux aussi se nourrissent des fruits du koto. Les chasseurs affirment que la nuit, ils abattent des herbivores tels que les biches et les gazelles sous ces arbres.

\section{III-3-fonctions économiques et matérielles}

L'Agni Bini a le foutou d'igname comme élément essentiel de son régime alimentaire. La confection de ce mets nécessite l'utilisation de mortiers et pilons. L'Iroko et le Tchimi sont des arbres qui résistent aux termites et qui sont très solides. Les bûcherons s'en servent pour fabriquer des pilons et des mortiers. Ils utilisent également leur tronc pour fabriquer des cuvettes et des cuillères en bois. Ils en fabriquent en grande quantité. Ces produits artisanaux sont commercialisés sur le marché à des fins économiques. Ils sont aussi utilisés dans la fabrication de chaises traditionnelles pour hommes et pour femmes qu'ils vendent également. Ces chaises sont achetées par des chefs de familles et les femmes âgées. Ils se doivent d'en avoir dans les biens qu'ils lègueront en héritage à leur progéniture. Le cas échéant ce serait un déshonneur pour la famille. Le tronc des fromagers sert à la confection de pirogues pour traverser la rivière en saison de crue.Le fromager et l'iroko, compte tenu de leur grande taille, jouent pour les paysans un rôle de marqueur d'espace dans les jachères. Leur ombrage et leurs contreforts (celui du fromager) servent également de lieu de repos et de partage des repas aux heures de pause et d'abri en saison de pluie. Notons qu'une fois asséchées, ces plantes constituent pour les femmes de la population enquêtée du bois de chauffe. Cependant, ces ressources ligneuses ne servent pas d'énergies fossiles aux familles pour lesquelles elles sont totémiques. Et, ce au risque de voir émerger dans ces ménages des épidémies et des malédictions inexplicables par toute personne dépourvue de puissances mystiques.Par ailleurs, c'est sous l'arbre walè que les paysans ensemencent les tubercules d'ignames les plus valorisées. En effet, les populations se représentent cet arbre comme germant toujours sur des sols fertiles où l'abondante reproduction des cultures n'a 
jamais fait défaut. L'exemple du '‘tambi', variété d'igname précoce très valorisée, est exigé pour satisfaire aux cérémonies nuptiales. Le vieux Kouassi. K ; explique cette pratique en ces termes : «les ignames telles que le tambi et kponan sont des variétés représentant une forte valeur symbolique chez nous. En offrir à un bienfaiteur ou un hôte est signe de considération et de respect. Pour nous rassurer d'en disposer dans notre grenier à la prochaine récolte, nous les cultivons sous le walè. Cet arbre ne nous trompe jamais ».

\section{III-4-Fonctions religieuses et mystiques}

Le fromager (gnanhan) est un arbre foncièrement mystique. En effet, avant la création d'un futur village, il faut en trouver sur place un fromager qui servirait à la longue aux chefs de terre pour l'immolation des animaux en guise de sacrifice. Les contreforts de cet arbre constituent un sanctuaire pour protéger les attaques mystiques et des épidémies éventuelles contre le village. Si l'on n'en trouve pas sur place, le chef de terre coupe une branche d'un autre fromager et la plante à la place dénommée Asiè ti sou. Dans chaque village Bini une telle place existe. C'est sert de lieu d'immolation pour des sacrifices visant à réparer une offense contre la terre et l'esprit des ancêtres. Ces arbres sont aussi le lieu de résidence des génies protecteurs de la nature; les abattre serait synonyme de les déloger et s'attirer de fait des malédictions et des malheurs provenant des esprits et de la terre.

Aussi, faut-il noter que lorsqu'une femme n'enfante pas et qu'elle consulte un devin, ce dernier peut lui requérir un cabri ou un coq, à la mesure des difficultés à surmonter. Celle-ci offrira en sacrifice par le biais du chef de terre un de ces animaux sous l'iroko ou le 'tchimi'" pour lui permettre d'enfanter. Les génies procurent en plus, des richesses (or) sous ces arbres protégés.Les espaces sacrés tels que l'assiédou n'zoué bâh (il y existe à la fois une rivière et une forêt sacrées) situé entre Kouassi-Datékro et Yakassé-Bini est très redoutés. A l'occasion de cette étude, tous les enquêtés ont refusé de nous y accompagner. Les mânes qui habitent ce site n'autorisent que les seuls initiés à le visiter. Ce massif forestier abriterait les ancêtres disparus des deux villages. Parfois les populations de Yakassé prétendent entendre les nuits des rythmes de tam-tam et des chants biens connus des deux villages. Habituellement, au lendemain d'une nuit festive ou même de deuil ; à l'occasion d'une rencontre inopinée entre acteurs des deux villages (ils se fréquentent), ils se félicitent comme de coutume de l'originalité des chants et des rythmes. Il arrive parfois que les uns et les autres se surprennent d'être congratulés. Et ce dans la mesure où, en vérité, ils ne se rappellent pas avoir organisé de danse de réjouissance encore moins funèbre pour mériter des encouragements des pairs du village voisin.En effet, dans leur entendement, les populations deYakassé pensent que ces chants, rythmes et danses entendus la nuit passée ; avaient eu lieu à Kouassi-Datékro et vice-versa. Tout ceci pour dire qu'il arrive des moments où les "ancêtres"' disparus, locataires de ces lieux, festoient et se réjouissent dans le monde. Il faut toutefois noter que pour ces deux villages voisins, si les chants ont une connotation funèbre, c'est le signe qu'un prestigieux personnage (chef ou roi malade) de l'un des deux villages disparaitra les deux semaines à venir.

Le loyebooh (forêt de la guerre) d'Abondji est une forêt protectrice des espèces. Elle peut être fréquentée par tous (Hommes et animaux) à la seule condition de se sentir en danger. Selon l'enquêté Kouassi. M ; «Le loyebooh n'a jamais été brulé par le feu de brousse qui à l'époque se pratiquait chaque année. C'est le lieu de refuge de toute être vivant qui se sent en danger. Si un chasseur, ou même son chien poursuit un animal et que celui-ci entre dans cette forêt, Celui-ci se doit arrêter de le suivre. S'il s'entête et qu'il y entre, il s'y perdra pendant plusieurs jours et seuls les devins pourront le retrouver. Aussi, il en sortira toujours grièvement blessé. Cependant, si un individu se trouve en danger et qu'il y pénètre, la forêt le protège. C'est ainsi que quand la guerre contre Samori Touré survînt, et que la défaite du groupe se profilait à l'horizon, le chef a demandé que tout le village se refugie dans le loyebooh pour se prémunir des attaques de l'ennemi. La population est sortie de cette forêt sans que son intégrité physique soit atteinte. L'ennemi s'en était définitivement parti ».

\section{III-5- Fonctions de régulation sociale}

L'organisation sociale des villages des sous-préfectures de Kouassi-Datékro et de Boahia est identique. A la tête de chaque village se trouve un chef dont l'une des fonctions est de veiller à la protection de l'environnement. Laquelle fonction est déléguée au chef de terre de chaque village. Ce dernier veille sur le respect des jours dits ''noirs', jour de repos est obligatoire et la protection des espèces et espaces sacrés. Toute profanation des sites protégés occasionne forcement une réparation (offrandes sacrificielles) que le chef de terre exige du contrevenant. Nul ne peut ni ne doit refuser de se soumettre à cette disposition. Si tel est le cas, c'est le chef de famille qui doit contraindre le membre fautif à y adhérer. Si celui-ci refuse d'offrir les sacrifices, c'est le chef de famille lui-même qui offre les éléments exigés par le chef de terre. Ensuite, au sein de la famille cette situation se règle selon le principe de la matrilinéarité. En effet, l'obligation est faite à l'oncle de l'individu indélicat de restituer au chef de cette famille les offrandes déjà cédées au maître de la terre.

En clair, chez le peuple Agni-Bini, plusieurs familles constituent chaque village. Chaque famille a un chef «Awoulo Kpangni». Il est l'autorité morale de la famille et est responsable devant le chef du village. Quand un membre de la grande famille pose un acte contre les réglementations du village, il interpelle de prime abord 
l'oncle et le cas échéant le géniteur de ce dernier qui est tenu à son tour d'amener son neveu ou son fils à fournir les éléments de sacrifices qu'exige l'ampleur de son acte. Ne pas se soumettre aux injonctions de son oncle ou de son père est un acte grave chez le peuple Bini et nécessite une peine de cent (100) coups de fouet en présence des membres de la grande famille sans exclusion. La peine peut aller jusqu'à l'exclusion de ce membre de la famille. Une telle exclusion est également accompagnée de malédictions.

Il convient de noter que l'arrivée des colons dans cette localité a occasionné l'introduction de l'école et des religions révélées. Cette situation ne va pas sans apporter de profondes mutations dans les habitudes des populations.

\section{DISCUSSION}

L'étude a montré que chez les Agni-Bini plusieurs mécanismes ont été mises en place pour protéger les espèces et espaces. La protection des ressources naturelles par les populations locales a fait l'objet d'études que la littérature ne cesse de relever. Dans ce sens, certains auteurs pensent comme il a été signifié aussi cette étude que le modèle endogène de gestion des ressources laisse supposer que l'interdiction d'accès à des ilots forestiers, des espèces végétales et des animaux se fait dans le but de favoriser la reproduction des ressources. Dugast(2002) soutient que le mode d'exploitation du milieu par certaines sociétés vise à limiter la charge de ponction sur l'environnement. Cette préoccupation est plus motivée par le souci d'assurer le renouvellement des ressources sur lesquelles repose la substance que par celui de préserver en lui-même. Cette étude a aussi montré que des efforts sont actuellement encore consentis pour le renouvellement des ressources dans le but d'une pérennisation des activités des ascendants. Dans cette logique de conservation du patrimoine, les jeunes des sous-préfectures de Kouassi-Datékro et de Boahia, singulièrement les élèves et étudiants organisent chaque année des séances de reboisement. Même si l'action de la jeunesse a une portée économique, elle vise aussi à la pérennisation des espèces des dites localités. C'est une manière d'être en conformité avec les mânes protecteurs. Raison pour laquelle avant l'entame de cette activité de reboisement, les élèves et étudiants reçoivent l'onction des anciens à l'occasion d'une cérémonie solennelle de libation au lancement de ce programme. Par ailleurs, d'autres auteurs ont relevé que la cohésion sociale n'est rien d'autre que l'emprise des savoirs écologique sur l'organisation sociale qu'il convient d'appréhender comme intimement liée à la sphère sociale. C'est pourquoi, les transgressions des systèmes de gestion des ressources impactent significativement la cohésion sociale. Ainsi, «les représentations culturelles des phénomènes environnementaux ne sont pas aisément séparables de ce que font les populations. Elles varient en conséquence selon le contexte et les opinions les concernant, selon la perception de ceux qui les utilisent à des fins différentes. En ce sens, tout aspect particulier de l'environnement constitue l'objet de multiples expériences et de multiples savoirs ». ( Fairhead et Leach, 1994). De ce qui précède, on en déduit que, pour l'autochtone, la nature et le social sont deux entités certes matériellement dissociées mais demeurent symboliquement en interaction réciproque. Le respect des règles de la nature et des principes de ses locataires influencent la cohésion sociale et vice-versa. Ce lien entre l'homme et la nature est renforcée par la présence des forces invisibles qui habitent l'écosystème naturel tel que nous l'avons évoqué dans la présente étude. Dans cette perspective, (Duchesne, 2000), parlant des représentations de l'univers écologique par les Agni écrit que : «le système de croyances anyi, le monde naturel (terrestre et aquatique) appartient à des créatures invisibles envoyées par Dieu-Ciel (appelé Nyamien), les boson. Les boson habitent les sommets remarquables du paysage (collines, montagnes, rochers), les eaux (courantes ou dormantes), certains grands arbres et des portions de forêt non dégradées. Habituellement, la communauté villageoise dans son ensemble est placée sous l'égide d'un boson localisé dans la nature et auquel est rendu un culte». Ces représentations liées à la nature favorisent la conservation de la biodiversité à travers les multiples fonctions, les valeurs socioculturelles et les richesses matérielles et immatérielles que les autochtones attribuent aux ressources de leur environnement. Ces valeurs héritées des ancêtres datant de l'origine de la création du village continuent de se perpétuer. Dans le cas de cette étude, la perpétuation de ces valeurs se poursuit à travers l'action concomitante des populations locales et sous-préfectorale. L'autorité du sous-préfet s'étend à tous les domaines d'activités et aussi dans la protection de l'environnement où il en est le garant. Aidé dans sa tâche par les agents des eaux et forêts et les autorités villageoises, il sanctionne tous les auteurs des feux de brousse, l'exploitation abusive des ressources ligneuses et protège dans la même mesure les essences rares. Il est la seule autorité à délivrer dans la sous-préfecture les permis d'exploitation des essences et vérifie sa validité. Ces actions concourent à la préservation des héritages qui sont l'ensemble des espaces et espèces laissés par les ancêtres. Cet héritage transmis aux générations présentes suscite chez ceux-ci les sens de la mesure et une attitude révérencieuse à l'occasion de la ponction des ressources. Il doit donc être géré dans le souci d'être légué aux générations futures. Ce mode de gestion raisonnée constitue un autre argument militant en faveur de la volonté manifeste des autochtones de protéger leur milieu naturel. La protection du milieu naturel n'est plus seulement le fait des autochtones résidents dans un milieu dont ils réclament l'appartenance. Des acteurs exogènes interviennent à travers des lois qui établissent la réglementation du patrimoine naturel de la nation. Telles sont les aires protégés, les flores et faunes que le gouvernement de la Côte d'Ivoire a pris soin sous le 
regard du Ministère de l'Environnement et des Eaux et Forêts à travers ses agents de sauvegarder. Toutes activités de chasse, de pêche, d'exploitation des ressources sont interdites dans le souci de protéger ses espaces et espèces pour le bien de la nation et des générations futures.

\section{CONCLUSION}

Cette étude sur les pratiques endogènes de conservation et de protection de la biodiversité fait ressortir qu'il existe de nombreux espaces et espèces et dans l'Est de la Côte d'ivoire en pays Agni.Vu ces fonctions (fonction médicinale, fonction économique et matérielle, fonction alimentaire, fonction religieuse, fonction mystique...), ce nombre important d'espèces et d'espaces constitue une richesse pour le peuple agni. Ils représentent un univers naturel qui ne doit pas être spolié. Cet univers en pays agni-Bini est un lieu sacré induit d'une force surnaturelle pour le pèlerinage des populations désireuses améliorer leurs conditions de vie sociales. Le rapport que les populations Agni-Bini entretiennent avec cette force pourrait attirer sur l'individu et sa communauté soit la bienveillance (respect des coutumes) soit la sévérité (non-respect de la tradition) de celui qui est la source de toute vie ; DIEU être suprême et invisible mais immanent par le biais de dieux secondaires : génies, ancêtres. Il est donc clair que, l'idéologie religieuse traditionnelle chez les Agni-Bini, conçue sur une alliance individu-nature ou même communauté-nature, une alliance étroite entre les dieux et la nature est le fondement de la protection de l'environnement. La subsistance de cette alliance confirme donc une relation de symbiose entre l'Agni-Bini et son patrimoine naturel qu'il préserve.

\section{BIBLIOGRAPHIE}

[1] Boutrais, J.,(2002). Patrimoine animal et territoire chez des sociétés peules, patrimonialiser la nature tropicale dynamiques locales, enjeux internationaux, IRD, éditions collection colloques et séminaires, Paris, pp 167-188.

[2] Duchesne, V., (2000). Le Bossonisme ou comment être « moderne et de religion africaine, Présence africaine, $\mathrm{n}^{\circ} 161 / 162$, pp.299-314.

[3] Dugast, S.,(2002). Modes d'appréhension de la nature et gestion patrimoniale du milieu, patrimonialiser la nature tropicale dynamiques locales, enjeux internationaux. Paris :IRD, éditions collection colloques et séminaires, pp 31-78

[4] Fairhead, J., et Leach, M.,(1994). Représentation culturelle africaine et gestion de l'environnement, politique africaine $N^{\circ} 53$, l'homme et la nature en Afrique, OSTROM, Paris.

[5] IBO, J (2004). "Savoirs naturalistes paysans et protection de nature en Côte d'Ivoire », Annales de l'Université Abdou Moumouni. IRD. Niamey. pp140-158. 